\title{
Alphabet Permutation for Differentially Encoding Text
}

\author{
Gad M. Landau \\ Ofer Levi \\ University of Haifa \\ Steven Skiena* \\ SUNY Stony Brook
}

May 21, 2004

\section{Introduction}

One degree of freedom which is usually not exploited in developing high-performance textprocessing algorithms is the encoding of the underlying atomic character set. Typically, standard character encodings such as ASCII or Unicode are assumed to be a fixed fact of nature, and indeed for most classical string algorithms the assignment of exactly which symbol maps to which $k$-length bit pattern appears to be an issue of no consequence.

In this paper, however, we consider a text compression method where the specific character set collating-sequence employed in encoding the text has a big impact on performance. We demonstrate that permuting the standard character collating-sequences yields a small win on Asian-language texts over gzip. We also show improved compression with our method for English texts, although not by enough to beat standard compression methods. However, we also design a class of artificial languages on which our method clearly beats gzip, often by an order of magnitude.

The significance of this work lies partially in evaluating an interesting approach to text compression. Even more, however, we seek to raise awareness of character encodings in the string-algorithms community and ask the question whether alphabet-permutation can lead to improvements in other string and text-processing algorithms.

\section{Differential Encoding}

Differential coding is a common preprocessing step for compressing numerical data associated with sampled signals and other time series streams. The temporal coherence of such signals

${ }^{*}$ Corresponding author: skiena@cs.sunysb.edu 
implies that the value at time $t_{i}$ likely differs little from that at $t_{i+1}$. Thus representing the signal as an initial value followed a stream of difference (i.e. $t_{i+1}-t_{i}$ for $0 \leq i<n$ ) should consist primarily of small differences. Such streams should be more compressible using standard techniques like run-length encoding, Huffman coding, and gzip than the original data stream.

Here we consider differential encoding of text by treating each character code as an integer. By taking the differences modulo the size of the alphabet, we can ensure that they can always be encoded using the same number of bits as the original character symbols.

Under what conditions might such a differentially encoded text $T^{\prime}$ be more compressible than the original straight text $T$ ? Let $w=s_{1} s_{2} \ldots s_{k}$ be a string of length $k$ which occurs multiple times in $T$. We note that $w^{\prime}=\delta_{1} \delta_{2} \ldots \delta_{k-1}$ occurs the same number of times in $T^{\prime}$, where $\delta_{i}=s_{i+1}-s_{i}$. Since $w^{\prime}$ is shorter by one character that $w$, differential encoding might seem inherently counter-productive to the goals of higher compression.

However, there are two potential benefits. First, the string $w^{\prime}$ in $T^{\prime}$ may arise from several different strings within $T$, whenever the strings have a common shift pattern. A well-known example is the collision of the suffixes of "IBM" and "HAL" in a differential encoding using the ASCII collating sequence [5]. Second, with the proper collating sequence we would expect to have a skewing in the distribution of symbols toward those representing smaller differences.

It is impossible to tell a priori whether differential encoding with alphabet permutation will lead to improved compression on any given language. For this reason, we report experimental results in the sections to follow.

The most relevant previous work is [3], where alphabet permutation was employed to improve the performance of compression algorithms based on the Burrows-Wheeler transform [2]. Previous work on differential coding for text compression includes [8, 9]. Our work goes farther in our efforts to optimize the alphabet permutation, extending the results to Asian language encoding, and building a theory of languages for which differential coding will be effective.

\section{Experiments on English Texts}

The key to successful differential encoding of English (or any other class of languages) lies in identifying the best collating sequence. However, designing the optimal character permutation is a non-trivial problem. In principle, we seek the order which most frequently collapses popular substrings into identical sequences of differences. However, this criteria is not well-defined and does not lend itself to local improvement-based optimization.

Instead, we seek an ordering which minimizes the expected size of the differences. Such an ordering would be expected to skew the distribution of symbols towards those representing small differences, which would clearly improve the performance of zeroth-order entropy compression algorithms such as Huffman codes. We would also expect that repeated difference sequences would occur more frequently in collating sequences which lead to a skewed symbol distribution. 


\begin{tabular}{l|rrr|rr|rr} 
& \multicolumn{3}{|c}{ Original } & \multicolumn{2}{c}{ Differential } & \multicolumn{2}{r}{ Permuted-Diff } \\
file & size & gzip & huffman & gzip & huffman & gzip & huffman \\
\hline book1 & 767476 & 286727 & 434221 & 322455 & 489396 & 321347 & 472108 \\
book2 & 598615 & 182574 & 339493 & 203269 & 381969 & 202812 & 369285 \\
paper1 & 51093 & 15709 & 28896 & 17600 & 32500 & 17548 & 31447 \\
paper2 & 81860 & 27375 & 46724 & 30440 & 52328 & 30350 & 50508 \\
paper3 & 45997 & 16464 & 26395 & 18519 & 29575 & 18467 & 28565 \\
paper4 & 13032 & 4826 & 7556 & 5435 & 8455 & 5397 & 8211 \\
paper5 & 11730 & 4236 & 6660 & 4824 & 7508 & 4800 & 7304 \\
paper6 & 36483 & 10942 & 20096 & 12219 & 22950 & 12169 & 22200 \\
news & 365318 & 118124 & 207541 & 133529 & 231233 & 133192 & 227751 \\
gesture & 44893 & 14910 & 25160 & 16756 & 28541 & 16717 & 27673 \\
bib & 104812 & 28833 & 59255 & 31834 & 66380 & 31694 & 65815 \\
trans & 73923 & 12734 & 39746 & 14227 & 45292 & 14207 & 44844 \\
progc & 38348 & 10760 & 20187 & 11948 & 23086 & 11920 & 22795 \\
progp & 48266 & 9290 & 24440 & 10414 & 27954 & 10400 & 27694 \\
progl & 71213 & 14620 & 36878 & 16230 & 42168 & 16209 & 41759 \\
12sad10 & 424342 & 154122 & 237963 & 173374 & 270706 & 172830 & 260760 \\
\hline
\end{tabular}

Table 1: Effect of Optimized Permutation on Differential Coding Methods.

Therefore, we seek the circular $n$-permutation $\pi$ which minimizes the objective function

$$
\min _{\pi \in \Pi} \sum_{i=1}^{n} \sum_{j=1}^{n} d(i, j) p\left(\sigma_{i}, \sigma_{j}\right)
$$

where $p(i, j)$ is the probability that symbol $j$ immediately follows symbol $i$, i.e. $p(i, j)=$ $P(j \mid i)$, and $d(i, j)$ is the shortest "distance" from $i$ to $j$ around the circular permutation. Thus $d(i, j)=\min (|j-i|, n-|j-i|)$. This is an instance of the notorious quadratic assignment problem, an optimization problem significantly harder in practice than the traveling salesman problem [1]. If unweighted by probabilities, permutation optimization is related to the linear assignment problem [4], which although NP-complete under very restrictive conditions is managable in practice through heuristics [7]. Alterate optimization criteria are no doubt possible, but this is the one we used.

To estimate the conditional character-probabilities for the optimized collating sequence for English text, we used letter-pair (bigram) frequencies derived from a large corpus of text analyzed in [12], including the famous Brown corpus. The Discropt [10, 11] system was run for 10 hours optimizing the permutation over these frequencies, resulting in the following collating sequence:

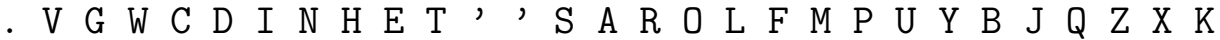

Table 1 compares differential compression using both the standard and optimized collating sequence, with both standard Huffman codes and gzip employed for encoding. The 


\begin{tabular}{lr|rr|rr} 
& Original & \multicolumn{2}{c}{ Permuted } & \multicolumn{2}{c}{ Permuted-Diff } \\
file & size & gzip & huffman & gzip & huffman \\
\hline book1 & 768260 & 303884 & 394003 & 347211 & 466801 \\
book2 & 599399 & 194201 & 313925 & 221163 & 362138 \\
paper1 & 51877 & 16751 & 27181 & 19084 & 30970 \\
paper2 & 82644 & 28902 & 42112 & 32848 & 49727 \\
paper3 & 46781 & 17471 & 24037 & 19900 & 28040 \\
paper4 & 13816 & 5086 & 6888 & 5764 & 8061 \\
paper5 & 12514 & 4576 & 6402 & 5186 & 7093 \\
paper6 & 37267 & 11652 & 19294 & 13251 & 21734 \\
news & 366102 & 127102 & 207326 & 144729 & 224090 \\
gesture & 45677 & 15884 & 23738 & 18241 & 26992 \\
bib & 105596 & 30372 & 58164 & 34151 & 65152 \\
trans & 74707 & 13762 & 42218 & 15465 & 45317 \\
progc & 39132 & 11641 & 21428 & 13242 & 22926 \\
progp & 49050 & 10205 & 26732 & 11589 & 28067 \\
progl & 71997 & 15796 & 39840 & 17839 & 42033 \\
12sad10 & 425126 & 164272 & 220261 & 188444 & 257470 \\
\hline
\end{tabular}

Table 2: Effect of Elias Predictive Coding on Differential Coding Methods.

permuted collating sequence typically reduces the size of the Huffman-encoded differential sequences by $3-4 \%$, and gzip-encoded differential sequences by about $1 \%$ - however, both encoding algorithms work substantially better on the original text instead of the differential text.

The use of a single fixed collating sequence for all characters does not effectively capture the second-order entropy of the language, because the symbol distribution following each letter of the alphabet is distinct. In Table 2, we identify the best symbol permutation following each character, for each file separately; a method akin to a static version of Elias predictive coding [6]. Such a method produces better compression rates for differential encoding on sufficiently large files, even with the cost of storing the character permutation matrix. Interestingly, although Huffman codes work better on the Elias coded files, gzip does significantly worse than on the original file.

\section{Experiments on Asian-Language Texts}

We reasoned that differential encoding might perform better on Asian-language texts, because the larger size of the alphabet makes such texts more closely resemble quantized signals. However, accurately measuring the character bigram frequencies in Asian-language texts is made difficult by the enormous size of the alphabet. Vast amounts of training text would be needed to estimate the $\left(2^{16}\right)^{2}$ bigram-pairs of 16-bit UNICODE. Further, quadratic 
assignment problems of such size are intractable to solve.

For this reason, we chose a different method to construct the alphabet permutation for 16-bit UNICODE. We assume that symbol usage in any fixed-length alphabet obeys a Zipf's law-type distribution, so frequently-used symbols are much more popular than expectation. Our permutation was derived by determining the symbol frequencies for all characters over all documents, and ordering the symbols in order of decreasing frequency. Since the most popular symbols are located near each other in the code space, we would anticipate that such an ordering would lead to smaller average differences over arbitrary encodings.

Table 3 details the results of our experiments on Chinese, Japanese, and Korean UNICODE texts. We experimented with both 8-bit and 16-bit recoded alphabets. The 8-bit alphabet permutation produced worse results than the original alphabet encoding for both gzip and Huffman codes, but permuting the full 16-bit alphabet encoding did permit the differential gzip encoding to beat the conventional gzip encodings by $1-2 \%$ on almost all files.

\section{Experiments on Martian-Language Texts}

To demonstrate that gzip can be significantly beaten via differential encoding for certain languages, we define a class of artificial languages which we will call Martian.

Martian words evolve in families. Each family is defined by a length- $(l-1)$ sequence of differences from 0 to $\alpha-1$, where $\alpha=|\Sigma|$ and $\Sigma$ is the length of the alphabet. There are $\alpha$ distinct length- $l$ words in each family, formed by prepending each $\sigma \in \Sigma$ to the difference sequence. For example, for $\Sigma=\{a, \ldots, z\}$ the family $(+2,+3,-6)$ defines the words acfz, $b d g a, c e h b$, and so forth.

In the experiments below, we compress randomly generated Martian texts constructed with the following parameters:

- The alphabet size is $\alpha$, ranging from 2 to 256 .

- The number of word families is $f$. Word families were generated by sampling uniformly at random from the $\alpha$ possible differences. No effort was made to ensure that words would be part of at most one family.

- The length of each each word is $l$.

- The number of words in the text is $n$. The random text was generated by sampling uniformly with replacement from the $\alpha f$ words in the language.

We achieve our greatest improvement in differentially encoding Martian texts with relatively short texts drawn from large families of long words. Table 4 demonstrates that differential encoded gzip results in 5.8 times better compression than plaintext gzip on files from 2500 to 50,000 words for 20 families of 20-character words. Even more extreme performance is obtainable by further lengthening the words. 


\begin{tabular}{|c|c|c|c|c|c|c|c|c|}
\hline \multirow[b]{2}{*}{ file } & \multicolumn{3}{|c|}{ Original } & \multicolumn{2}{|c|}{ 8-bit encoding } & \multicolumn{3}{|c|}{ 16-bit encoding } \\
\hline & size & gzip & huff & diff-gzip & diff-huff & huff & diff-huff & perm-gzip \\
\hline ChuanXiLu1 & 100904 & 30545 & 57307 & 32774 & 73389 & 33828 & 54734 & 29953 \\
\hline ChuanXiLu2 & 129750 & 39753 & 73804 & 42908 & 94946 & 43794 & 70785 & 39054 \\
\hline ChuanXiLu3 & 96894 & 30314 & 55210 & 32782 & 70839 & 33577 & 53859 & 29779 \\
\hline dai & 83100 & 40318 & 64540 & 51850 & 70611 & 41203 & 73290 & 38544 \\
\hline RenXue & 229590 & 76878 & 130902 & 83277 & 167918 & 78475 & 126092 & 75810 \\
\hline XinMinShuo & 357138 & 115668 & 202585 & 125114 & 259954 & 118312 & 190772 & 114354 \\
\hline Xunzi & 88094 & 46182 & 69361 & 58050 & 76014 & 46089 & 82352 & 44296 \\
\hline ZhengMeng & 66212 & 35033 & 51465 & 44044 & 56086 & 34586 & 62659 & 33165 \\
\hline ZhouDunyiJi & 85646 & 26487 & 48782 & 28778 & 62153 & 29773 & 48315 & 25968 \\
\hline ZhuziYulei1-6 & 297888 & 87767 & 167904 & 93602 & 216643 & 95531 & 156285 & 86801 \\
\hline ZhuziYulei14-18 & 446066 & 129448 & 252811 & 137478 & 325836 & 140393 & 231406 & 128122 \\
\hline ZhuziYulei7-13 & 273836 & 85480 & 155937 & 91431 & 199607 & 89439 & 145787 & 84477 \\
\hline Gan & 156494 & 68335 & 106217 & 87897 & 117985 & 78332 & 114737 & 67514 \\
\hline Goju-no-to & 114108 & 60709 & 83634 & 77433 & 91324 & 64086 & 98553 & 59781 \\
\hline Hojoki & 22936 & 10779 & 15127 & 13595 & 16861 & 12357 & 17786 & 10626 \\
\hline horoki & 420774 & 166957 & 277984 & 211118 & 294482 & 193615 & 264312 & 165275 \\
\hline Jigokuhen & 58226 & 24460 & 39741 & 31198 & 43952 & 30591 & 45156 & 24083 \\
\hline Kageronikki & 202632 & 76612 & 112690 & 100539 & 129172 & 80347 & 106842 & 76135 \\
\hline kaidoki & 68158 & 31979 & 46631 & 39928 & 50387 & 36185 & 55050 & 31270 \\
\hline KanadehonChushingura & 164122 & 63883 & 110045 & 79724 & 115361 & 74324 & 111094 & 62601 \\
\hline Kappa & 84092 & 32524 & 57883 & 41222 & 62053 & 43587 & 61638 & 32031 \\
\hline Kokoro & 339050 & 137499 & 233746 & 178618 & 256945 & 163798 & 232484 & 135906 \\
\hline KoshokuGoninOnna & 82240 & 41057 & 57557 & 52177 & 63581 & 43006 & 67447 & 40119 \\
\hline KoshokuIchidaiOnna & 125278 & 56263 & 86343 & 71393 & 95161 & 63396 & 96308 & 55020 \\
\hline Makura-no-soshi & 276342 & 117992 & 178011 & 154409 & 199284 & 126817 & 179134 & 116691 \\
\hline Midaregami & 45768 & 15051 & 26696 & 19039 & 27827 & 18506 & 27133 & 14719 \\
\hline Monogatari & 63878 & 21782 & 36172 & 28288 & 40890 & 24498 & 33777 & 21615 \\
\hline MurasakiShikibu-nikki & 77972 & 33616 & 51395 & 43627 & 57087 & 37939 & 55137 & 33280 \\
\hline OkuNoHosomichi & 31430 & 15878 & 22434 & 19329 & 24147 & 18299 & 28551 & 15453 \\
\hline SankaWakashu & 178138 & 60699 & 107901 & 78642 & 114954 & 70872 & 101243 & 59562 \\
\hline Shayo & 207568 & 78946 & 133906 & 101544 & 147648 & 96732 & 133419 & 78025 \\
\hline SonezakiShinju & 41170 & 17357 & 28388 & 21461 & 29981 & 21693 & 32848 & 17008 \\
\hline Taketori & 40762 & 17643 & 27875 & 22832 & 30886 & 21292 & 32271 & 17444 \\
\hline Tsurezuregusa & 145156 & 62583 & 95940 & 80163 & 106215 & 69703 & 101209 & 61560 \\
\hline UgetsuMonogatari & 102562 & 52121 & 70229 & 65919 & 78896 & 55063 & 82895 & 51204 \\
\hline Ukigumo & 259698 & 114305 & 182091 & 145127 & 196343 & 132205 & 186899 & 112534 \\
\hline $\mathrm{cjk}$ & 51172 & 25178 & 41410 & 31152 & 44671 & 28878 & 51207 & 24377 \\
\hline Ijangui & 22240 & 13667 & 18431 & 16459 & 19854 & 14902 & 27781 & 13097 \\
\hline zuochuan-sjis & 95666 & 34710 & 54191 & 45093 & 60316 & 38822 & 48472 & 34872 \\
\hline
\end{tabular}

Table 3: Effect of Optimized Permutation on Differential Coding Methods, for Chinese, Japanese, and Korean Texts 


\begin{tabular}{|c|c|c|c|c|c|c|c|c|c|c|}
\hline \multirow[b]{2}{*}{$f$} & \multirow[b]{2}{*}{$l$} & \multirow[b]{2}{*}{ size } & \multicolumn{2}{|c|}{$\alpha=32$} & \multicolumn{2}{|c|}{$\alpha=64$} & \multicolumn{2}{|c|}{$\alpha=128$} & \multicolumn{2}{|c|}{$\alpha=256$} \\
\hline & & & gzip & diff-gzip & gzip & diff-gzip & gzip & diff-gzip & gzip & diff-gzip \\
\hline 5 & 5 & 2500 & 4584 & 3687 & 5174 & 4169 & 6445 & 4607 & 8606 & 5132 \\
\hline 5 & 5 & 5000 & 9059 & 7229 & 9680 & 8162 & 11080 & 8948 & 14061 & 9958 \\
\hline 5 & 5 & 10000 & 18000 & 14259 & 18879 & 16144 & 20371 & 17604 & 23782 & 19391 \\
\hline 5 & 5 & 25000 & 44110 & 35131 & 45993 & 39929 & 47751 & 43458 & 52756 & 47395 \\
\hline 5 & 5 & 50000 & 87358 & 69896 & 90828 & 79579 & 92981 & 86511 & 100849 & 93925 \\
\hline 5 & 10 & 2500 & 5607 & 4171 & 6745 & 4601 & 9672 & 5021 & 14498 & 5500 \\
\hline 5 & 10 & 5000 & 10435 & 8155 & 11380 & 8983 & 14691 & 9776 & 21855 & 10647 \\
\hline 5 & 10 & 10000 & 19860 & 16030 & 20645 & 17691 & 24505 & 19193 & 35454 & 20823 \\
\hline 5 & 10 & 25000 & 47754 & 39603 & 48407 & 43767 & 53932 & 47344 & 76321 & 51168 \\
\hline 5 & 10 & 50000 & 94066 & 78884 & 94701 & 87275 & 103071 & 94267 & 144064 & 101737 \\
\hline 5 & 20 & 2500 & 7184 & 4814 & 10184 & 5351 & 16414 & 5761 & 27385 & 6154 \\
\hline 5 & 20 & 5000 & 12253 & 9358 & 15792 & 10492 & 25493 & 11308 & 46488 & 12032 \\
\hline 5 & 20 & 10000 & 22375 & 18447 & 26880 & 20742 & 43485 & 22351 & 84336 & 23768 \\
\hline 5 & 20 & 25000 & 52726 & 45644 & 60333 & 51458 & 97928 & 55429 & 198693 & 58950 \\
\hline 5 & 20 & 50000 & 103345 & 90982 & 115986 & 102755 & 188780 & 110577 & 389213 & 117645 \\
\hline 10 & 5 & 2500 & 5132 & 4244 & 6114 & 4602 & 7875 & 5115 & 10165 & 5537 \\
\hline 10 & 5 & 5000 & 9851 & 8292 & 10859 & 8940 & 13329 & 9893 & 17403 & 10849 \\
\hline 10 & 5 & 10000 & 19630 & 16402 & 20437 & 17574 & 23174 & 19340 & 29305 & 21153 \\
\hline 10 & 5 & 25000 & 48429 & 40476 & 48646 & 43384 & 52418 & 47383 & 63399 & 51613 \\
\hline 10 & 5 & 50000 & 96041 & 80643 & 95232 & 86327 & 100784 & 94021 & 120058 & 102085 \\
\hline 10 & 10 & 2500 & 6529 & 4616 & 8917 & 5024 & 13038 & 5497 & 18429 & 5931 \\
\hline 10 & 10 & 5000 & 11344 & 8987 & 13972 & 9745 & 20137 & 10610 & 30120 & 11525 \\
\hline 10 & 10 & 10000 & 20992 & 17638 & 23915 & 19099 & 33315 & 20734 & 52755 & 22497 \\
\hline 10 & 10 & 000 & 018 & 489 & 3776 & 47051 & 72654 & 50927 & 120689 & 55366 \\
\hline 10 & 10 & 50000 & 98363 & 86607 & 103531 & 93611 & 138149 & 101239 & 233663 & 110140 \\
\hline 10 & 20 & 2500 & 9534 & 5347 & 26 & 5791 & 24297 & 6194 & 36224 & 6637 \\
\hline 10 & 20 & 5000 & 15188 & 52 & 255 & 11236 & 41580 & 11991 & 65932 & 12830 \\
\hline 10 & 20 & 10000 & 26404 & 20328 & 40508 & 22077 & 76069 & 23561 & 126013 & 25224 \\
\hline 10 & 20 & 25000 & 59834 & 50240 & 92024 & 54594 & 180190 & 58263 & 305697 & 62485 \\
\hline 10 & 20 & 50000 & 115555 & 100143 & 177859 & 108741 & 353604 & 116089 & 605099 & 124485 \\
\hline 20 & 5 & 2500 & 5836 & 4741 & 7204 & 5113 & 9137 & 5542 & 11250 & 5948 \\
\hline 20 & 5 & 5000 & 755 & 9214 & 12646 & 9885 & 16049 & 10771 & 20421 & 11656 \\
\hline 20 & 5 & 10000 & 20740 & 18123 & 22688 & 19295 & 27742 & 20972 & 36038 & 22835 \\
\hline 20 & 5 & 25000 & 50222 & 44628 & 52318 & 47263 & 61265 & 51167 & 81557 & 55802 \\
\hline 20 & 5 & 50000 & 98754 & 88708 & 101124 & 93778 & 117056 & 101301 & 157107 & 110648 \\
\hline 20 & 10 & 2500 & 8352 & 5134 & 11695 & 5536 & 16410 & 5965 & 21301 & 6376 \\
\hline 20 & 10 & 5000 & 3582 & 899 & 18602 & 10619 & 27224 & 11486 & 37950 & 12365 \\
\hline 20 & 10 & 10000 & 23701 & 19293 & 31375 & 20613 & 48249 & 22294 & 70462 & 24247 \\
\hline 20 & 10 & 25000 & 54079 & 47374 & 69759 & 50462 & 110791 & 54630 & 168058 & 59676 \\
\hline 20 & 10 & 50000 & 104742 & 93830 & 133662 & 100145 & 215310 & 108519 & 330452 & 118582 \\
\hline 20 & 20 & 2500 & 13564 & 5888 & 21528 & 6312 & 31968 & 6745 & 42379 & 7198 \\
\hline 20 & 20 & 5000 & 21703 & 11312 & 37090 & 12108 & 58743 & 12929 & 81159 & 13791 \\
\hline 20 & 20 & 10000 & 38051 & 22116 & 68420 & 23649 & 111898 & 25271 & 158012 & 26970 \\
\hline 20 & 20 & 25000 & 87213 & 54422 & 161892 & 58257 & 271528 & 62320 & 389073 & 66646 \\
\hline 20 & 20 & 50000 & 168542 & 108284 & 318406 & 115932 & 538072 & 124003 & 775298 & 132668 \\
\hline
\end{tabular}

Table 4: Comparing gzip and Differential-gzip Compression of Martian Language Texts, as a Function of Family Size, Word Length, and Text Length. 
Differential gzip outperforms normal gzip because the coherence of words in a family is unrecognizable by gzip until each word has been seen enough times to be encoded by gzip as a single symbol. The advantage of differential gzip on Martian texts will disappear once all words in each family are defined by code strings, but this takes much longer with the plaintext gzip.

Experiments with several random permutations of the Martian alphabet confirms that there is no benefit from differential encoding unless the correct character ordering is used. Determining the optimal ordering (or even a good one) from a text corpus is presumably an intractable problem. This leaves the theoretical (but highly unlikely) possibility that English text can be efficiently differentially compressible were only the correct alphabet permutation were found.

\section{Acknowledgments}

We thank Danny Keren for his insight that coding techniques for quantized signals can be applied to strings, Dafna Sheinwald for helpful discussions, and Vihnthuy Phan and Pavel Sumazin for optimizing the English-alphabet permutation using Discropt.

\section{References}

[1] R. Burkard, E. Cela, P. Pardalos, and L. Pitsoulis. The Quadratic Assignment Problems, pages 241-238. Kluwer, 1998.

[2] M. Burrows and D. Wheeler. A block-sorting lossless data compression algorithm. Digital Systems Research Center Research Report 124, 1994.

[3] B. Chapin and S. Tate. Higher compression from the burrows-wheeler transform by modified sorting. In IEEE Data Compression Conference, 1998.

[4] P. Chinn, J. Chvátolvá, A. K. Dewdney, and N. E. Gibbs. The bandwidth problem for graphs and matrices - a survey. J. Graph Theory, 6:223-254, 1982.

[5] A. C. Clarke. 2001: A Space Odyssey. Roc, reissue edition, 2001.

[6] P. Elias. Interval and recency rank source coding: two on-line adaptive variable-length schemes. IEEE Trans. on Information Theory, 33:3-10, 1987.

[7] N. Gibbs, W. Poole, and P. Stockmeyer. A comparison of several bandwidth and profile reduction algorithms. ACM Trans. Math. Software, 2:322-330, 1976.

[8] D. Gottlieb, S. Agerth, P. Lehot, and H. Rabinowitz. A classification of compression methods and their usefulness for a large data processing center. National Computer Conference, 44:453-458, 1975. 
[9] M. F. Lynch. Compression of bibliographic files using an adaptation of run-length coding. Information Storage and Retrieval, 9:207-214, 1973.

[10] V. Phan and S. Skiena. An improved time-sensitive metaheuristic optimizer. In 3rd Int. Workshop on Experimental and Efficient Algorithms (WEA), Buzios, Brazil, 2004.

[11] V. Phan, S. Skiena, and P. Sumazin. A time-sensitive system for black-box combinatorial optimization. In Workshop on Algorithm Engineering and Experimenation (ALENEX '02), 2002.

[12] H. Rau and S. Skiena. Dialing for documents: an experiment in information theory. Journal of Visual Languages and Computing, pages 79-95, 1996. 